\title{
Origin of Ferromagnetism in Perovskite Cobaltites
}

Takayoshi Kishida, ${ }^{1,2}$ Stephen J. Pennycook, ${ }^{2,3}$ and Matthew F. Chisholm ${ }^{2}$

${ }^{1}$ Department of Technology Group, Analysis \& Simulation Center, Asahi Kasei Corporation, 2-1 Samejima, Fuji, Shizuoka, 410-8501, Japan

2 Materials Science and Technology Division, Oak Ridge National Laboratory, Oak Ridge, TN 37831, USA

${ }^{3}$ Department of Materials Science and Engineering, University of Tennessee, Knoxville, Tennessee 37996, USA

Transition metal perovskite oxides exhibit various distributions of charge, spin and orbital order. In perovskite cobaltites, the spin degree of freedom resulting from $\mathrm{Co} 3+$ ions distinguishes them from manganites and cuprates. Many questions remain about the magnetic ordering in these systems. A recent investigation using first-principles calculations suggested that room temperature ferromagnetism in $\mathrm{Sr}_{3} \mathrm{YCo}_{4} \mathrm{O}_{10+\delta}(\mathrm{SYCO})$ arises through the formation of 'spin bags' [1].

We have used a fifth-order aberration-corrected Nion UltraSTEM200 scanning transmission electron microscope to investigate the local atomic structure and Co spin state in SYCO, a room-temperature ferromagnetic metal, and $\mathrm{SrCoO}_{2.5}$ (SCO), an antiferromagnetic insulator. Both materials consist of two kinds of layers with nearly perovskite structure that alternate along the $a$-axis of SCO, and the $c$ axis of SYCO. Oxygen vacancies are present and ordered in one layer while the second layer is fully oxygenated. Ferromagnetism in SYCO is expected to depend sensitively on the spin state of the Co atoms, the layer of ordered oxygen vacancies and the presence and order of the $\mathrm{Y}$ atoms on the $\mathrm{Sr}$ sub-lattice. [1-4]. We have used annular bright-field (ABF) imaging [5,6] to locate oxygen columns and determine the oxygen octahedral tilts in these materials. High-angle annular dark-field (HAADF) imaging is used to detect the presence of cation displacements in SYCO and the layers containing ordered oxygen vacancies in both materials. Electron energy-loss spectroscopy (EELS) is used to probe local changes in the oxygen $K$-edge and Co $L$-edge that reflect variations in spin state.

Typical ADF and ABF images from SCO are seen in Figure 1 along with image simulations based on the multi-slice method. The images were obtained simultaneously at $200 \mathrm{kV}$ using a probe convergence angle of $30 \mathrm{mrad}$. The presence and location of the ordered oxygen vacancies is easily seen in the ABF images along with the local distortions of the cation and anion sub-lattices. The ADF images also reveal the local distortions in the cation sub-lattices. Figure 2 shows the structure of SYCO, and the simulation reveals the local distortions in the cation sub-lattices along with the Y ordering. EEL spectra obtained from adjacent cobalt-oxygen planes reveal variations in the Co $L$ edge and $\mathrm{O} K$-edge in SCO (Figure 3). Such features in cobaltite EEL data give information on Co spin state [7], and attempts to directly map the 'spin bags' in SYCO will be presented [8].

\section{References}

[1] JL Bettis Jr et al., Chem. Mater 24 (2012) p. 3117.

[2] W Kobayashi et al., Phys. Rev. B 72 (2005) 104408.

[3] I Terasaki, Physica. B 383 (2006) p. 107.

[4] H Nakao et al., J. Phys. Soc. Jpn. 80 (2011) 023711.

[5] Okunishi, et al. Microsc. Microanal. 15 (2009), p. 164. 
[6] R. Ishikawa, et al. Nature. 10 (2011), p. 278

[7] J. Gazquez, et al. Nano Lett. 11 (2011), p. 973.

[8] This work was supported in part by the DOE Office of Basic Energy Sciences, Materials

Sciences and Engineering Division (S.J.P.,M.F.C.).

(a)

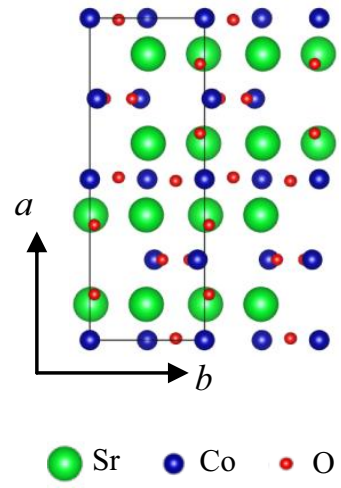

(b)

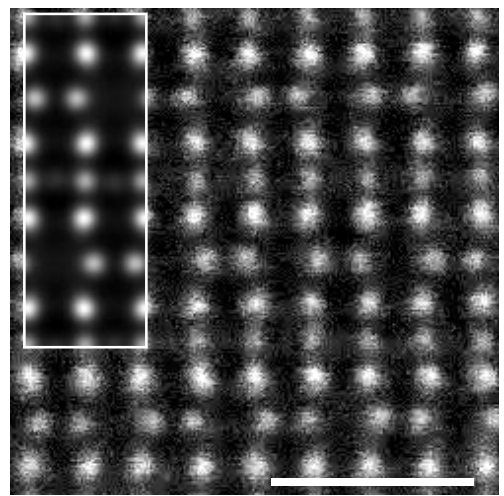

(c)

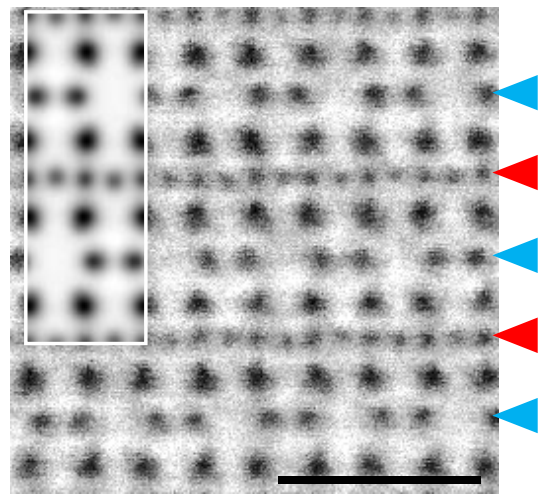

Figure 1. (a) Crystal structure of $\mathrm{SrCoO}_{2.5}$ viewed along the $c$ axis. (b) ADF image obtained with detector range 30-200 mrad, (c) ABF image obtained with detector range 15-30 mrad, ADF and ABF images were obtained simultaneously. Simulated images (HREM Inc.) using the structure of Fig. 1a are inset in the left side of each image. The blue and red arrowheads highlight the position of the ordered oxygen vacancies and the fully oxygenated planes. Scale bars: $1 \mathrm{~nm}$.

Figure 2. (a) Crystal structure of $\mathrm{Sr}_{4} \mathrm{YCo}_{3} \mathrm{O}_{10+\delta}$ viewed along the $b$ axis. (b) HAADF image obtained with detector ranges 60-200 mrad. Simulated images (HREM Inc.) using the structure of Fig.2a are inset in the left side of the image. The yellow lines highlight the distortions of atom column positions. Scale bar: $1 \mathrm{~nm}$. (a)

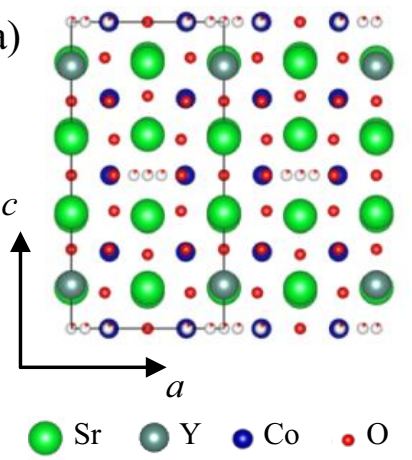

(b)

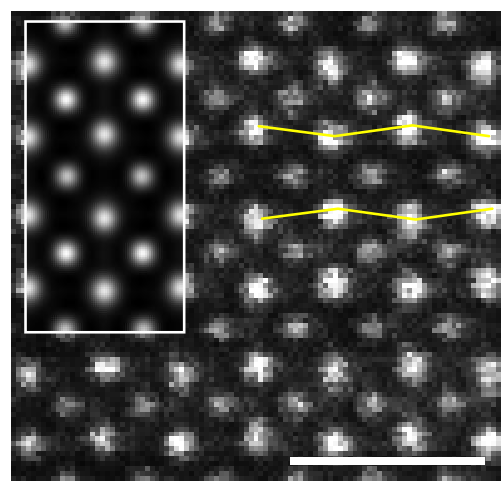

Figure 3. EEL spectra obtained from $\mathrm{SrCoO}_{2.5}$ viewed along the $c$ axis, showing the $\mathrm{O} K$ edge and the Co $L_{2,3}$ edge from alternating Co-O planes. The blue lines are from planes containing the oxygen vacancies and red lines are obtained from the fully oxygenated planes as seen on the right side of Fig. 1c.

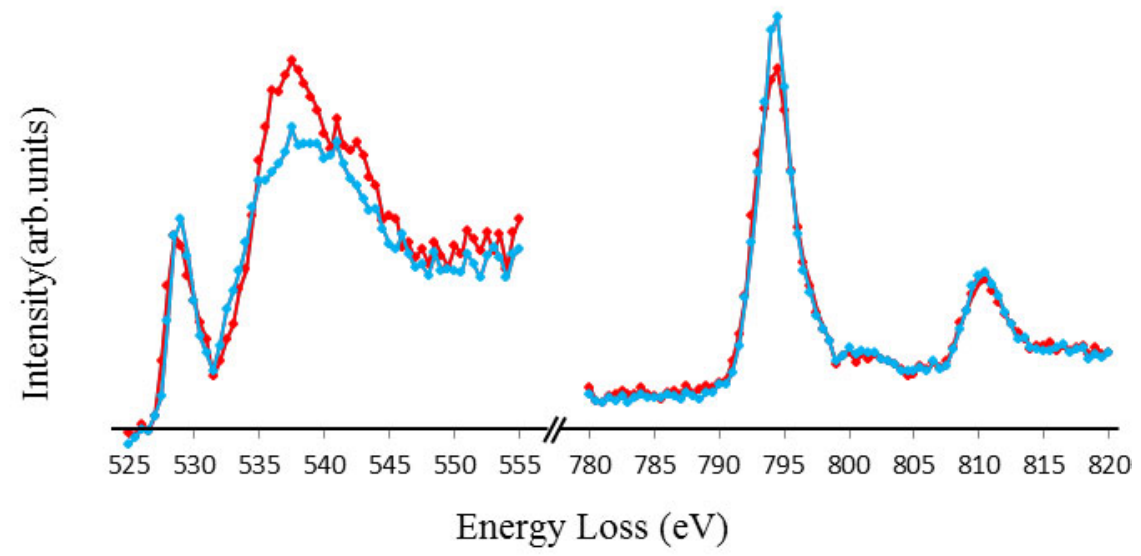

